\title{
Desenvolvimento de um Ambiente de Simulação ROS/Gazebo para Inspeção Fotogramétrica 3D de Risers com RPAS
}

\author{
Salazar J. ${ }^{* * * *}$ Regner D. * Oliveira D. H. * Marcellino G. * \\ Buschinelli P. ${ }^{*}$ Santos J. M. ${ }^{* *}$ Marinho C. ${ }^{* *}$ Pinto T. ${ }^{* * * * *}$ \\ * Labmetro - Dep. Eng. Mecânica - Univ. Federal de Santa Catarina \\ (UFSC) \\ ** CENPES/Petrobras, Rio de Janeiro \\ ***jose.salazar@labmetro.ufsc.br \\ **** tiago.pinto@ufsc.br
}

\begin{abstract}
The use of remotely piloted aircrafts (RPAs) for industrial inspection has grown a lot thanks to the latest developments in the related technologies and in embedded systems. In the oil and gas industry, RPAs can be of great value for the inspection of different types of structures and components. Among these are the flexible risers, pipes that perform an important role in offshore oil and gas exploration. The present work describes the development of a ROS/Gazebo simulation environment for 3D optical inspection of risers through photogrammetry. The simulations allow for multiple testing scenarios that would be expensive to be done on the field with varying types of acquisition strategies and sensors. Also presented is the 3D reconstruction base on images retrieved from simulations, as well as an analysis of part of a measured riser.

Resumo: A utilização de aeronaves remotamente pilotadas (RPAs) na inspeção industrial tem crescido muito graças aos últimos avanços das tecnologias envolvidas e dos sensores embarcados. $\mathrm{Na}$ indústria do petróleo e gás os RPAs podem ser de grande valia na inspeção de diversas estruturas e componentes. Dentre estas, os risers flexíveis, que são tubulações que cumprem um importante papel na exploração de petróleo e gás em alto mar. O presente trabalho descreve o desenvolvimento de um ambiente de simulação ROS (Robot Operating System) e Gazebo (a multi-robot simulator) para a inspeção óptica 3D de risers utilizando fotogrametria. As simulações permitem realizar inúmeros testes que seriam muito dispendiosos para serem executados em campo, como a variação de estratégias de aquisição e sensores. Apresenta-se também a reconstrução 3D a partir de imagens obtidas por simulação, assim como a análise de um dos trechos de risers medidos.
\end{abstract}

Keywords: RPA; risers; flexible pipes; photogrammetry; ROS/Gazebo.

Palavras-chaves: RPA; risers; tubo flexível; fotogrametria; ROS/Gazebo.

\section{INTRODUÇÃO}

Nos últimos anos, graças ao constante crescimento da microeletrônica e a eficiência computacional, a utilização de aeronaves pilotadas remotamente $\left(\mathrm{RPAs}^{1}\right)$, tem aumentado rapidamente em diversas áreas de aplicação, tais como a militar, segurança, engenharia civil, arqueologia, agronomia, silvicultura, geomática e telecomunicações (Shakhatreh et al., 2018). Dentre as atividades realizadas pode-se destacar o mapeamento aéreo, operações de resgate, explorações geofísicas, monitoramento de trafego e inspeção de infraestruturas tanto civis quanto industriais (Kridsada et al., 2016) (Jordan et al., 2018). A inspeção industrial visa a redução de três importantes fatores: risco humano, tempo e custo (Kridsada et al., 2016) (Shakhatreh et al., 2018) (Jordan et al., 2018). A maioria das pesquisas nesta área tem sido focadas na inspeção de

1 RPAs também conhecidas como veículos aéreos não tripulados (VANTS) ou informalmente como drones instalações elétricas, linhas de transmissão e distribuição de energia, pontes, edificações e turbinas eólicas (Jordan et al., 2018). O emprego de RPAs neste tipo de inspeção se deve ao fato da facilidade em operar em áreas de difícil acesso, além de que podem ser equipados com diferentes tipos de instrumentos, incluindo: câmeras de imagem de alta resolução, câmeras térmicas, sonares, sensores LiDAR (light detection and ranging) e detectores de radiação. Os dados obtidos podem ser processados posteriormente ou visualizados em tempo real remotamente pelo técnico de inspeção (Jordan et al., 2018) (Shakhatreh et al., 2018).

Em 2014, através do encontro internacional da OGP (International Association of Oil and Gas Producers), a comunidade de petróleo e gás anunciou seu interesse na utilização de RPAs em três grandes áreas de aplicação: HSE (health safety environment), segurança e o monitoramento de instalações e integridade de ativos (Mercuri et al., 2017). Nesse contexto, um dos ativos mais importantes nos sistemas de exploração de petróleo em alto mar (offshore), 
são os denominados tubos flexíveis, também conhecidos como risers flexíveis $^{2}$ (Bai and Bai, 2014). Os risers são tubulações que interligam as unidades de produção (plataformas ou navios) com os poços submarinos, realizando variadas funções, que incluem a passagem de petróleo e gás, cabeamentos e o envio de produtos químicos para o poço. Sua estrutura é composta por diversas camadas metálicas e plásticas, conforme ilustra a Figura 1.

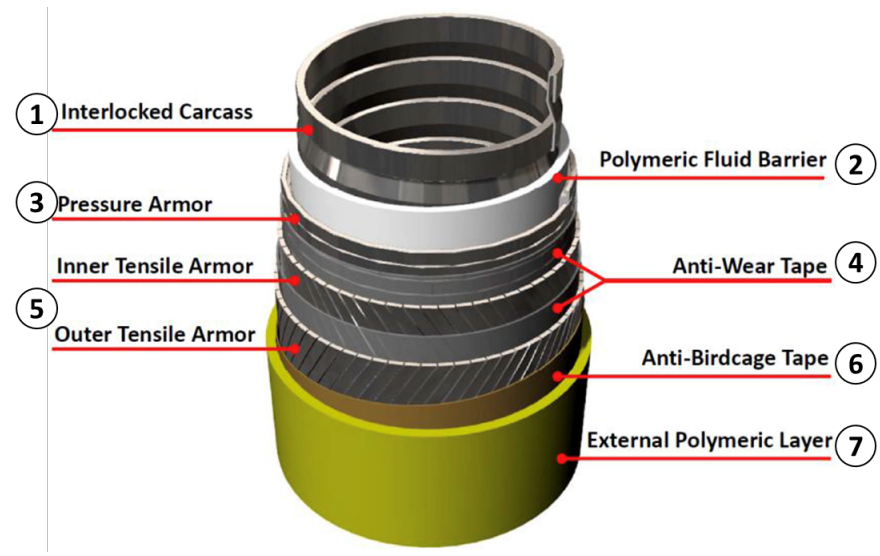

Figura 1. Estrutura típica de um riser flexível. 1) Carcaça de estrutura intertravada de aço; 2) Barreira polimérica interna; 3) Camada de aço resistente a pressão; 4) Fita anti desgaste; 5) Armadura de resistência a tensão; 6) Camada anti dilatação; (Li et al., 2019)

Estas estruturas operam em ambientes corrosivos, com condições ambientais extremas (Wang et al., 2016), o que aumenta o risco de falhas estruturais e mecânicas. Além do próprio ambiente, existem uma série de outros fatores que podem gerar danos estruturais ao risers, incluindo desgastes ou rasgos da capa externa devido ao atrito com outras estruturas durante o transporte, instalação ou até mesmo com cabos de embarcações ancoradas clandestinamente na plataforma; ruptura da armadura de resistência, entre outros (Marinho et al., 2006).

A falha de um riser pode gerar graves consequências econômicas e ambientais, razão pela qual devem ser feitas revisões periódicas a fim de manter a integridade dos dutos e toda sua instrumentação (Wang et al., 2016).

Tradicionalmente a inspeção de risers é realizada utilizando escalada industrial. O profissional encarregado realiza aquisições manuais, como captura de imagens e medição do diâmetro do duto (Figura 2). Estas operações requerem uma grande mobilização de recursos como equipamentos e estruturas auxiliares. É um processo demorado, onde inspeciona-se aproximadamente um riser por dia. Considerando um número típico de 50 risers em uma plataforma, a inspeção pode levar quase dois meses. Isto implica em muito tempo de ocupação dos funcionários na embarcação, que são limitadas, resultando em um alto custo operacional para a empresa contratante. Além de toda mobilização, tempo necessário e custos, estas operações possuem um alto risco para os profissionais, pois são realizadas em suspensão sobre o mar a grandes alturas, podendo chegar a mais de $20 \mathrm{~m}$.

2 Por simplicidade, risers flexíveis serão aqui simplesmente denominados de risers.

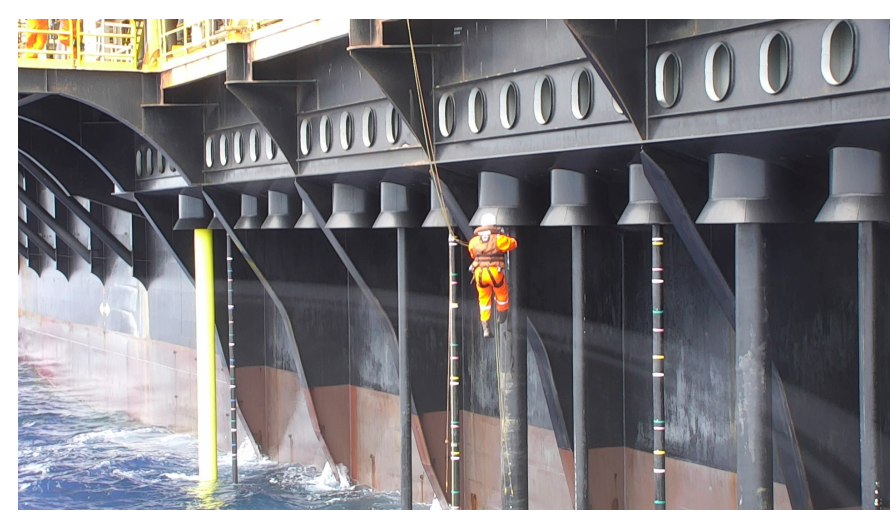

Figura 2. Inspeção de risers com escalada industrial.

Nesse contexto, a inspeção por meio de RPAs possibilitaria a realização da atividade de forma relativamente rápida e econômica, convertendo-se assim, em uma alternativa potencial para prevenção de falhas, avaliando o estado da estrutura externa e instrumentação, reduzindo drasticamente os riscos de segurança, tempo de inspeção e custos envolvidos. Recentemente RPAs estão sendo testados para a realização de inspeção visual de risers com a intenção de diminuir a necessidade de escaladores, os quais poderiam ser direcionados para pontos críticos previamente detectados pelas imagens obtidas com o RPA.

Mesmo com a aquisição de imagens com RPAs, os escaladores ainda são necessários para realizar atividades como medições geométricas manuais. Estas medições são muitas vezes feitas de forma precária devido a dificuldades relacionadas ao comprimento elevado do riser e do posicionamento do escalador. Com o objetivo de realizar medições geométricas a partir de inspeções com RPAs estão em desenvolvimento procedimentos e sensores adicionais para a medição geométrica 3D de risers.

Para o desenvolvimento de procedimentos de medição surge a necessidade de testar diferentes configurações para as trajetórias de aquisição que devem ser feitas pelo RPA, assim como a parametrização dos sensores embarcados, antes de que o hardware seja levado efetivamente a campo. A realização destes testes requer uma série de fatores, como a disponibilidade do hardware em si (RPA, sensores); tempo de voo do RPA; objetos simulando risers ou então ida à plataforma, que é uma atividade de alto custo, complexa e de demorada logística. Como solução, os ambientes de simulação surgem sendo uma excelente alternativa, já que permitem configurar e testar o sistema para executar procedimentos em diferentes condições sem os custos e riscos envolvidos dos testes em campo ou até mesmo em ambiente controlado em laboratório.

O presente trabalho relata o desenvolvimento de um ambiente virtual para simulação de diferentes configurações das trajetórias, inspeção visual e hardware na aquisição de dados para a inspeção da geometria $3 \mathrm{D}$ de risers. O ambiente de simulação é baseado no simulador Gazebo e no framework de código aberto ROS (Robotic operative system), que permite ao desenvolvedor criar pacotes para interagir com sensores, atuadores, etc, através de um sistema editor/assinante para a comunicação entre eles (Olivares-Mendez et al., 2014b). Por fim, tendo como base as imagens obtidas pela câmera nas diferentes trajetórias, 
é feita uma reconstrução 3D através de fotogrametria e o resultados obtidos com diferentes configurações podem ser comparados e avaliados.

\section{AMBIENTE DE SIMULAÇÃO}

O ROS provê um framework open source para desenvolver pacotes interativos com sensores reais, atuadores, robôs, etc., através de "tópicos" utilizando um mecanismo de publicação(publisher) / assinatura(subscriber) que permite uma comunicação estruturada entre eles. Além disso, é possível realizar a comunicação entre pacotes desenvolvidos e já existentes, aumentando assim seu potencial (Lara et al., 2017) (Olivares-Mendez et al., 2014a). Já o simulador Gazebo, fornece ferramentas para o desenvolvimento de robôs, sensores, diferentes condições ambientais como gravidade, vento, sol, chuva, neblina, etc., e um conjunto de plugins que facilitam o desenvolvimento da simulação. Uma das principais vantagens de utilizar este software, é que todos os pacotes (algoritmos, controle, etc) do ambiente virtual podem ser utilizados, com pequenos ajustes, em simulações reais com RPAs (Yoonseok Pyo, Hancheol Cho, Leon Jung, 2017) (Olivares-Mendez et al., 2014a). No trabalho desenvolvido realizou-se a simulação de uma câmera digital, considerando seus principais parâmetros, como: comprimento focal, resolução, frequência de aquisição e distância de captura. Também foram criadas trajetórias através de plugins no Gazebo para simular a movimentação do RPA no ambiente. Neste ponto cabe dizer que não foi considerado o modelo cinemático do RPA, que deverá ser realizado em trabalhos futuros.

\subsection{Geometria do cenário}

A partir da analise do ambiente físico tipico para realizar inspeções de risers em plataformas de exploração de petróleo em alto mar e considerando o paradigma de simulações para validar sistemas de robótica móvel, foi desenvolvido um cenário de simulação com características físicas do ambiente tais como uma embarcação FPSO (Floating Production Storage and Offloading), balcão de risers (risers balcony) a superfície do mar, um modelo de iluminação direcional linear com sol e neblina, assim como algumas perturbações de vento. O resultado é ilustrado na Figura 3.

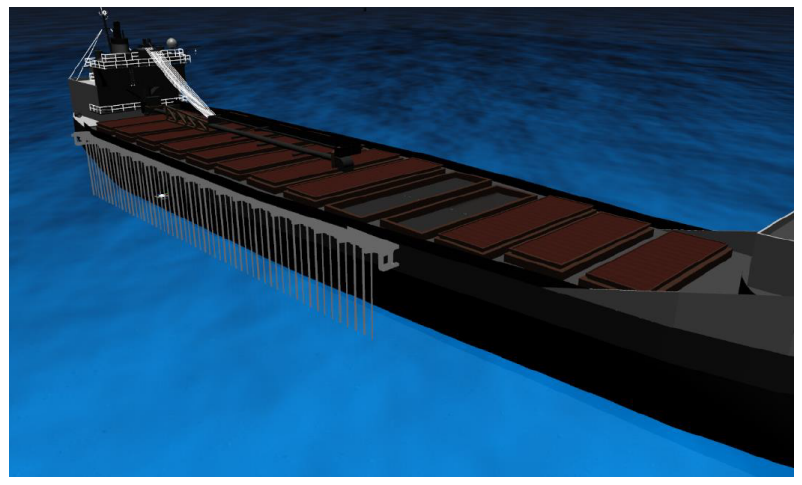

Figura 3. Simulação do ambiente de inspeção, incluindo a FPSO, balcão de risers e superfície do mar.

Todavia, devido a grande complexidade da cena, para a realização de testes de forma mais ágil a cena foi simplificada, resultando em um novo cenário cujo espaço físico é reduzido a cinco risers, casco da embarcação representado por uma parede espessa e parte do balcão de risers, conforme ilustra a Figura 4.

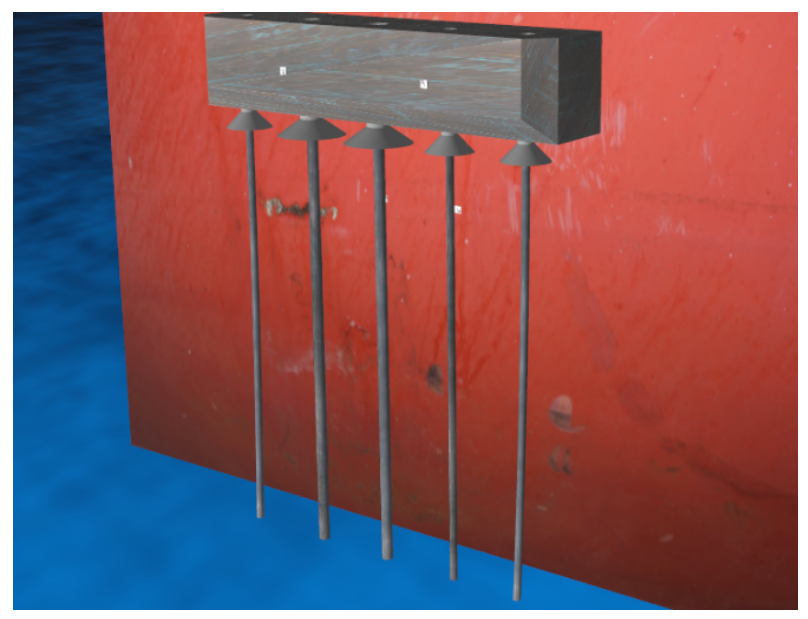

Figura 4. Cena simplificada contendo 5 risers.

\subsection{Geometria do mensurando (riser)}

Os risers foram modelados com o software AutoDesk Inventor, onde foram adicionadas algumas variações de geometria que podem ser encontradas em campo, como variações de diâmetro e outras deformações. Com o intuito de aumentar o realismo do ambiente e possibilitar a avaliação metrológica dos resultados foram otimizadas as malhas 3D (arquivos .stl) geradas no Inventor através do software de edição de malhas Meshlab. Para isto, realizouse o aumento do numero de vértices das malhas, passando de 9000 vértices a aproximadamente 95000 vértices, para depois realizar a reconstrução da superfície. Por fim, para adicionar textura aos modelos gerados, utilizou-se o software Blender, que é open source e suporta uma grande variedade de geometrias, incluindo as malhas de polígonos no formato COLLADA (Dere et al., 2010). Na Figura 5 é ilustrado o resultado final deste processo. Por fim, os modelos gerados são exportados para o Gazebo.
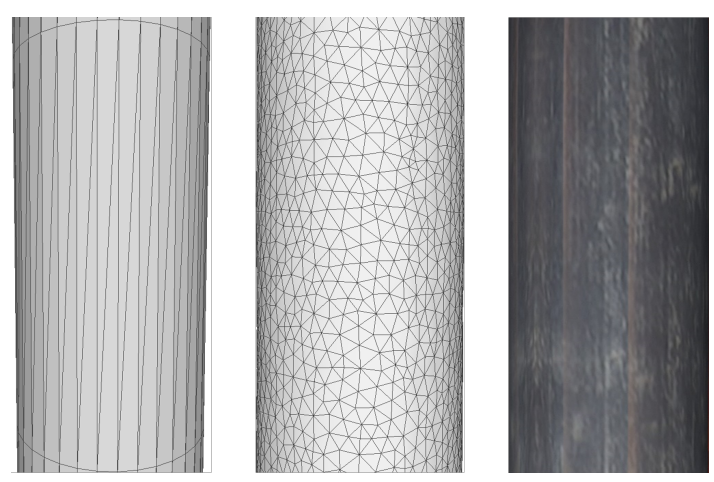

Figura 5. Modelagem da geometria e textura dos risers: (a) Modelo inventor (b) otimização Meshlab (c) textura blender 


\section{SIMULAÇÃO DA AQUISIÇÃO DAS IMAGENS}

Pode-se dividir o ambiente de simulação em três módulos principais: (i) Módulo da câmera, (ii) trajetória de captura e (iii) plugins para simulação. Uma descrição mais detalhada de cada módulo é feita a seguir.

\subsection{Modulo da câmera (sensor)}

O sensor de captura do ambiente de simulação foi desenvolvido através de um arquivo SDF (Simulation Description Format) em formato XML, onde são parametrizadas características reais da câmera e o tipo de lente a serem utilizados na simulação (Robotics Foundation, 2019). Considerou-se como base a câmera da DJI modelo X5S. Os principais parâmetros são:

- Horizontal fov (hfov): abertura do campo de visão horizontal em radianos. Seu valor pode ser obtido através de sua relação trigonométrica com a distância do ponto focal ao campo/plano de visão e o comprimento horizontal (largura) do campo de visão, conforme descrito na equação (1). A distância ao objeto (riser) foi definida em $5 \mathrm{~m}$ com base em requisitos de segurança. Já a largura do campo de visão foi definida como $3 \mathrm{~m}$, pois é possível visualizar mais de um riser simultaneamente, além de possibilitar uma certa tolerância na variação de posicionamento do RPA. Todavia, estes parâmetros poderão ser alterados futuramente, inclusive com base na análise de resultados obtidos em simulações. O valor resultante de hfov foi de $0,583 \mathrm{rad}\left(33,4^{\circ}\right)$. Apesar do tamanho do sensor não ser relevante na simulação, considerando-se a dimensão de um pixel de 3,4 um (sensor da X5S), o comprimento focal resultante é de $30 \mathrm{~mm}$.

$$
h f o v=2 \tan ^{-1}\left(\frac{\frac{1}{2} \text { largura campo de visão }}{\text { distância }}\right)
$$

- Resolução da imagem(width, height): resolução em pixels. As câmeras utilizadas nos ensaios possuem 5184 x 3456 pixels (18 Megapixel).

- Formato: determina o tipo de imagem a ser capturada. Definiu-se o padrão RGB (24 bits ou 8 bits por canal), descrito no SDF como R8G8B8.

Por fim, utilizou-se o plugin libgazebo_ros_camera.so para realizar a comunicação entre o sensor (câmera) e a plataforma ROS.

\subsection{Trajetória da câmera}

Com o intuito de analisar diferentes trajetórias para adquisição das imagens desenvolveu-se um plugin em $\mathrm{C}++$, o qual recebe coordenadas de orientação e posição escritas em um arquivo de texto, sendo possível produzir trajetos como ilustrado na Figura 6. Como a câmera é representada por um cubo estático, o movimento é desenvolvido por animação, deslocando o objeto no espaço tridimensional com a orientação desejada. Para isto foi utilizado um sensor IMU para leitura da posição atual e verificação do estado do objeto, o plugin foi desenvolvido com o conjunto de bibliotecas matemáticas de código aberto da Ignition Robotics (Robotics, 2019).

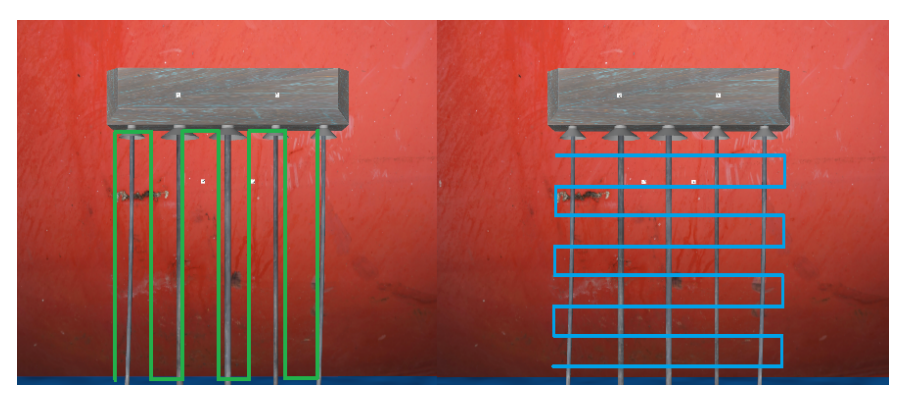

Figura 6. Exemplos de trajetórias para a aquisição de imagens.

\subsection{Captura das imagens}

A fotogrametria exige sobreposição entre imagens para a obtenção de bons resultados de medição. Um valor típico utilizado é a sobreposição em torno de $80 \%$ a $90 \%$. (Luhmann et al., 2006) (Li et al., 2018). Definiu-se assim uma combinação entre velocidade e período de aquisição que resulta em uma sobreposição mínima de $80 \%$. Para isso desenvolveu-se em C++ o nó "save_img_gazebo", que utiliza o pacote ROS image_transport com objetivo de assinar o tópico da câmera descrito na seção 3.1 e fazer a comunicação com o pacote CvBridge que converte as mensagens de imagem do ROS para imagens do OpenCV ${ }^{3}$. A Figura 7 ilustra a aquisição de uma imagem com o posicionamento da câmera no embiente de simulação 3D.

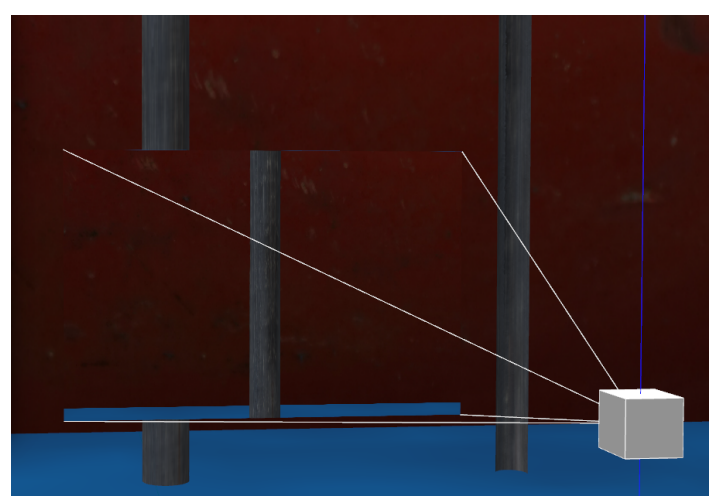

Figura 7. Captura de imagem no ambiente de simulação.

\section{RECONSTRUÇÃO 3D DO CENÁRIO E AVALIAÇÃO DOS RESULTADOS}

Uma vez finalizada a etapa de obtenção das imagens pela câmera, efetua-se a reconstrução tridimensional do ambiente de inspeção através da técnica de fotogrametria utilizando o software Metashape ${ }^{4}$. A fotogrametria é um método de medição sem contato capaz de transformar informações bidimensionais contidas em imagens em informações tridimensionais. A partir de duas ou mais imagens adquiridas de diferentes pontos de vista de um mesmo objeto é possível realizar sua reconstrução 3D. Além da alta sobreposição entre imagens citada anteriormente, existem outros parâmetros diretamente ligados à qualidade do resultado da reconstrução. Destaca-se aqui o GSD (Ground

\footnotetext{
3 Open Source Computer Vision Library. Site: https://opencv.org/ 4 Software Agisoft Metashape 1.5. Site: https://www.agisoft.com.
} 
Sample Distance), que representa a distância entre dois pixels projetados na cena, é a resolução do sistema, dado em unidade de comprimento por pixel. O GSD depende da resolução da câmera, da distância à cena e do comprimento focal da lente objetiva. Outros parâmetros de influência incluem: iluminação, heterogeneidade de cor da superfície do objeto e boa variação de pontos de vista. Para que seja possível obter informações de forma e localização espacial de cada ponto no espaço tridimensional é utilizado o princípio da triangulação, sendo os pontos 3D obtidos com base nos cruzamentos entre linhas projetadas a partir de pontos correspondentes nas imagens. Durante o processamento das imagens o software pode otimizar os parâmetros intrínsecos da câmera, como o comprimento focal e distorção da lente; assim como é capaz de obter a posição e orientação da câmera para cada imagem. Deve-se notar que a obtenção da estimativa inicial destes parâmetros antes do processamento pode reduzir muito o tempo da reconstrução 3D. Sua obtenção pode ser feita a partir de calibração da câmera e de dados do RPA. Mais detalhes sobre a técnica podem ser vistos em Luhmann et al. (2006). A seguir são expostos os resultados obtidos com o processamento e análise das imagens capturadas durante simulação.

\subsection{Reconstrução 3D da cena}

As imagens, que foram obtidas através da simulação de uma trajetória vertical, foram processadas no software Metashape. A seguir são listados os principais parâmetros do experimento.

- Imagens: 65

- Resolução: 5184 x 3456 pixels

- Pontos obtidos: 14.589 .000

- GSD: 0,734 mm/px

A Figura 8 mostra o resultado 3D obtido com o processamento fotogramétrico das imagens.

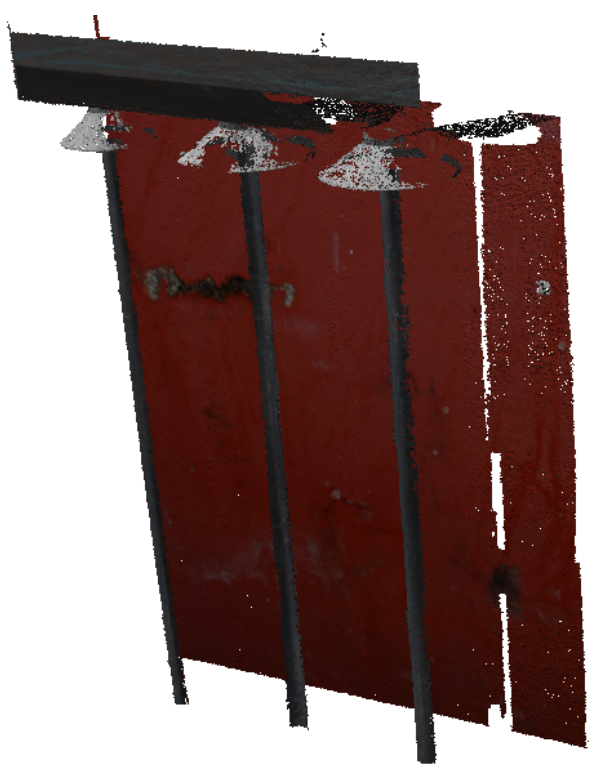

Figura 8. Nuvem de pontos 3D densa com textura obtida com o processamento das imagens capturadas na simulação.

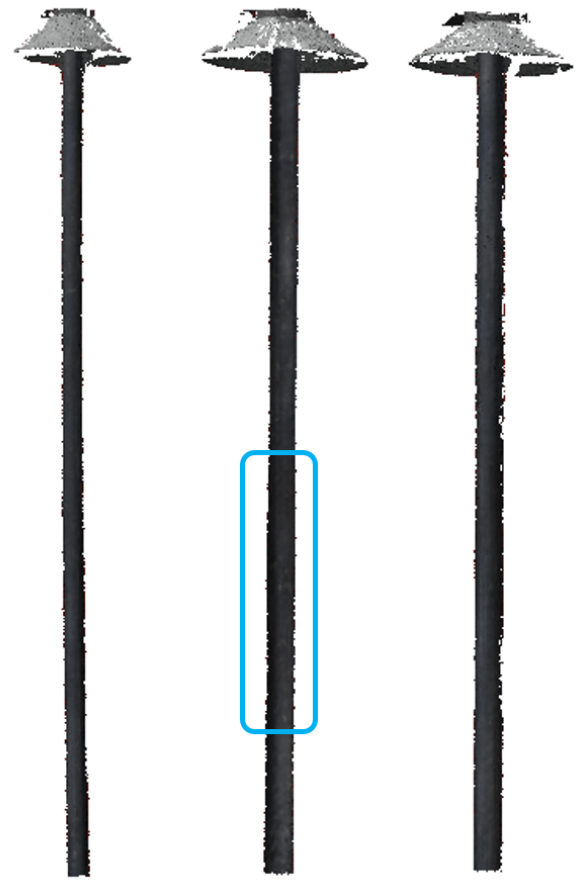

Figura 9. Risers segmentados para inspeção. Em azul o trecho do riser para analise

\subsection{Análise da medição de um trecho de riser}

A Figura 9 mostra três trechos de risers reconstruídos. A região destacada no trecho central foi comparada ao modelo CAD, resultando em um mapa de desvios, conforme ilustra a Figura 10. As cores do mapa de desvio representam a diferença/distância da medição ao CAD, sendo que este tipo de comparação permite avaliar a qualidade das medições realizadas e o impacto da variação de diferentes parâmetros (trajeto, resolução, entre outros) no resultado. Pela análise pode-se perceber que a medição está ruidosa, com variação de $\pm 6 \mathrm{~mm}$. Todavia, é importante destacar que este é um primeiro resultado de simulação, onde as texturas utilizadas, parâmetro da câmera e estratégias de medição ainda não foram otimizados.

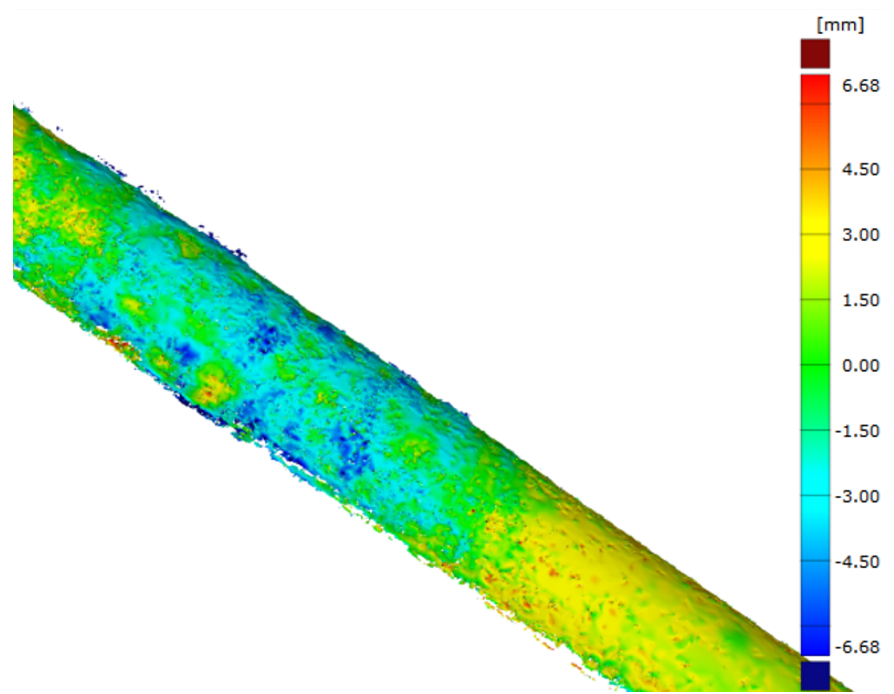

Figura 10. Mapa de desvios de um trecho do riser medido. 


\section{CONCLUSÃO}

O presente trabalho descreveu o desenvolvimento de um ambiente de simulação 3D utilizando ROS/Gazebo, onde é representada parte de uma plataforma de petróleo offshore. Uma câmera é deslocada no ambiente de simulação emulando aquisições realizadas por um sensor embarcado em um RPA. A simulação permitiu adquirir imagens para serem processadas em software fotogramétrico, resultando na reconstrução $3 \mathrm{D}$ da cena contendo os elementos de interesse (risers).

Uma sequência de 65 imagens dos risers, foram processadas em software de fotogrametria, que resultou em uma nuvem com mais de 14 milhões de pontos. O resultado 3D mostra que o trajeto simulado e textura utilizadas foram adequados para a fotogrametria. Realizou-se a análise de um trecho do riser medido, obtendo um mapa de desvios, onde pode-se notar claramente os defeitos no riser, ilustrando o tipo de resultado que poderá ser obtido com medições reais offshore.

Em trabalhos futuros será realizado o aprimoramento do ambiente de simulação, incluindo itens como um modelo de RPA, mais risers com diferentes defeitos, texturas com maior resolução e fidelidade, adição de ruido como neblina, pouco contraste, diferentes tipos de iluminação, assim como também ruido nos dados da IMU. Além disto, desenvolver biblioteca para a aquisição de imagens a partir de determinados deslocamentos assim como baseados na sobreposição das imagens. A simulação permitirá avaliar o impacto de diferentes parâmetros e procedimentos na qualidade da medição geométrica de interesse. Pretendese também simular um sistema de adquisição baseado em câmeras stereo para avaliar seu impacto no processo de reconstrução incluindo analise metrológica, avaliando a identificação de defeitos artificiais nos risers.

\section{AGRADECIMENTOS}

Os autores agradecem a Petrobras/CENPES pelo financiamento desta pesquisa através do Projeto VANT3D. Também agradecem a UFSC pela estrutura, ao CAPES pelas bolsas de Pós-graduação e por fim, à toda equipe VANT3D e engenheiros(as) da Petrobras que auxiliaram de alguma forma na realização do presente trabalho.

\section{REFERÊNCIAS}

Bai, Q. and Bai, Y. (2014). Flexible Pipe. In Subsea Pipeline Design, Analysis, and Installation, 559-578. Elsevier. doi:10.1016/B978-0-12-386888-6.00024-9.

Dere, S., Sahasrabudhe, S., and Iyer, S. (2010). Creating open source repository of $3 \mathrm{~d}$ models of laboratory equipments using blender. In 2010 International Conference on Technology for Education, 149-156. doi:10.1109/ T4E.2010.5550044.

Jordan, S., Moore, J., Hovet, S., Box, J., Perry, J., Kirsche, K., Lewis, D., and Tse, Z.T.H. (2018). State-of-the-art technologies for UAV inspections. IET Radar, Sonar E Navigation, 12(2), 151-164. doi:10.1049/iet-rsn.2017. 0251.

Kridsada, L., Chatchai, L., Manop, C., and Thana, S. (2016). Sustainability Through the Use of Unmanned
Aerial Vehicle for Aerial Plant Inspection. Offshore Technology Conference Asia. doi:10.4043/26576-MS.

Lara, A., Rego, B., Raffo, G., and Arias-Garcia, J. (2017). Desenvolvimento De Um Ambiente De Simulação De Vants Tilt-Rotor Para Testes De Estrategias De Controle. In XIII Simposio Brasileiro de Automaçao Inteligente, $2135-2141$.

Li, J., Huang, D., and Yang, P. (2018). Inspection method of images' overlap of UAV photogrammetry based on features matching. MATEC Web of Conferences, 173, 02022. doi:10.1051/matecconf/201817302022.

Li, X., Vaz, M.A., and Custódio, A.B. (2019). Analytical study of a repair methodology for flexible pipes antibirdcage tapes. Marine Structures, 63(October 2018), 289-303. doi:10.1016/j.marstruc.2018.10.001.

Luhmann, T., Robson, S., Kyle, S., and Harley, I. (2006). Close Range Photogrammetry - Principles, techniques and applications, volume 2. Whittles Publishing, Dunbeath. doi:10.1111/phor.12114. URL http://trid.trb. org/view . aspx?id=814766.

Marinho, M.G., dos Santos, J.M., and Carneval, R.d.O. (2006). Integrity Assessment and Repair Techniques of Flexible Risers. In Volume 4: Terry Jones Pipeline Technology; Ocean Space Utilization; CFD and VIV Symposium, volume 2006, 253-260. ASME. doi:10.1115/ OMAE2006-92467.

Mercuri, S.M., Fisicaro, A., and Tramontano, V. (2017). UAV the Impact \& Influence in the O\&G. Offshore Mediterranean Conference and Exhibition, 1-8.

Olivares-Mendez, M., Kannan, S., and Voos, H. (2014a). Setting up a testbed for uav vision based control using v-rep \& ros: A case study on aerial visual inspection. 447-458. doi:10.1109/ICUAS.2014.6842285.

Olivares-Mendez, M.A., Kannan, S., and Voos, H. (2014b). Setting up a testbed for UAV vision based control using V-REP \& ROS: A case study on aerial visual inspection. 2014 International Conference on Unmanned Aircraft Systems, ICUAS 2014 - Conference Proceedings, 447458. doi:10.1109/ICUAS.2014.6842285.

Robotics, O. (2019). What is Ignition Robotics? URL https://ignitionrobotics.org/docs/ all/overview $\{\backslash \#\}$ what-is-ignition-robotics-/.

Robotics Foundation, O.S. (2019). SDF Format. URL http://sdformat.org/.

Shakhatreh, H., Sawalmeh, A., Al-Fuqaha, A., Dou, Z., Almaita, E., Khalil, I., Othman, N.S., Khreishah, A., and Guizani, M. (2018). Unmanned Aerial Vehicles: A Survey on Civil Applications and Key Research Challenges. IEE Access, 1-58.

Wang, C., Shankar, K., and Morozov, E.V. (2016). Tailored design of top-tensioned composite risers for deepwater applications using three different approaches. Advances in Mechanical Engineering, 9(1), 1-18. doi:10. $1177 / 1687814016684271$.

Yoonseok Pyo, Hancheol Cho, Leon Jung, D.L. (2017). ROS Robot Programming (English). ROBOTIS Co.,Ltd, GeumCheon-gu, Seoul, Republic of Korea. doi:10.1109/ PROC.1983.12681. 